\title{
Advanced Analysis of Propagation Losses in Rectangular Waveguide Structures Using Perturbation of Boundary Conditions
}

\author{
Stephan Marini ${ }^{*}$, Michael Mattes ${ }^{*}$, Benito Gimeno ${ }^{\ddagger}$, Pablo Soto ${ }^{\S}$ and Vicente E. Boria ${ }^{\S}$
}

\# Departamento de Física, Ingeniería de Sistemas y Teoría de la Señal, Instituto Universitario de Física Aplicada a las Ciencias y Tecnologías, Universidad de Alicante, Spain * Laboratory of Electromagnetics and Acoustics, Ecole Polytechnique Fédérale de Lausanne, Switzerland

$\ddagger$ Departamento de Física Aplicada, Instituto de Ciencia de los Materiales, Universidad de Valencia, Spain

$\S$ Departamento de Comunicaciones, Instituto de Telecomunicaciones y Aplicaciones Multimedia, Universidad Politécnica de Valencia, Spain

\begin{abstract}
In this paper, propagation losses effects present in rectangular waveguide structures are rigorously considered. For this purpose, a new formulation based on the perturbation of the boundary conditions on the metallic walls of the waveguides combined with an Integral-Equation (IE) analysis technique is proposed. Following this advanced technique, the drawbacks of the classical power-loss method are overcome and a complex modal propagation constant is computed. To validate this theory, we have successfully compared our results with numerical data of lossy hollow waveguides. Next, a Computed-Aided-Design (CAD) software package based on such a novel modal analysis tool has been used to predict the propagation losses effects in a 2-pole symmetric bandpass filter.
\end{abstract}

Index Terms - Losses, Waveguide components, Dissipative filters, Complex propagation constant, Perturbation Method.

\section{INTRODUCTION}

New generation of telecommunication satellites are designed to operate at higher frequency bands and with more stringent filter characteristics to better exploit the available bandwidth. At higher frequencies, such as microwave and millimeter-wave bands, losses effects are more pronounced and can severely distort the electrical response of space hardware like filters and multiplexers. Therefore, the rigorous prediction of the ohmic losses effects affecting such passive waveguide devices is becoming more and more necessary [1][3].

Propagation losses in hollow waveguides have been typically solved by means of the classical perturbative power-loss method [4]. A very early contribution based on this method can be found in [5], where degenerated modes in lossy waveguides and cavities are treated. More recently rigorous consideration of metal losses in planar junctions has been included in [6] and [7], nevertheless losses associated with evanescent modes are not considered.

In this paper we propose an enhanced full-wave method for the computation of propagation losses in rectangular waveguide structures. This method, first proposed in [8] and [9], where only TM modes were treated with details, is based on the perturbation of the boundary conditions. As it will be demonstrated in the next sections, the proposed technique allows to overcome all the drawbacks of the classical powerloss method and it is valid assuming that the metallic walls are good conductors. In order to analyze realistic metalbased rectangular structures, we have included the proposed technique in a CAD tool based on the Integral Equation (IE) technique originally described in [10]. Proceeding in this way, a very precise modal analysis tool of rectangular waveguides filters considering propagation losses effects more accurately has been developed.

\section{THEORY}

To reduce the number of subscripts, we denote the electromagnetic field for perfectly conducting walls by a subscript zero, and the electromagnetic field for walls of finite conductivity value without subscript; moreover, we assume and omit the harmonic variation $e^{j \omega t}$ throughout this paper.

In a good conductor, the fields $\mathbf{E}$ and $\mathbf{H}$ are tangential to the surface and propagate normal to it. The small tangential electric field can be obtained using the Leontovich condition

$$
\mathbf{E}_{\|} \simeq Z_{s}\left(\hat{n} \times \mathbf{H}_{\mathbf{0} \|}\right)=\frac{(1+j)}{\sigma \delta}\left(\hat{n} \times \mathbf{H}_{\mathbf{0} \|}\right)
$$

where $\hat{n}$ is the unit normal vector outward from the conductor, $Z_{s}$ is the surface impedance, $\sigma$ is the metallic conductivity, $\delta=$ $\sqrt{2 /\left(\mu_{c} \omega \sigma\right)}$ is the skin depth, and $\mu_{c}$ the conductor magnetic permeability. The existence of this small tangential component of electric field, in addition to the normal electric field and tangential magnetic field, generates a power flow and ohmic loss along the walls of an uniform metallic waveguide with finite but large conductivity values

$$
\frac{d P}{d z}=-\frac{1}{2} \operatorname{Re}\left[\hat{n} \cdot \mathbf{E} \times \mathbf{H}^{*}\right]=\frac{1}{2 \sigma \delta} \oint\left|\hat{n} \times \mathbf{H}_{0 \|}\right|^{2}
$$


Equation (2) together with the conservation of energy are normally used in the classical pertubative power-loss method for the computation of the attenuation constant [4]

$$
\alpha=-\frac{1}{2 P} \frac{d P}{d z}
$$

where $-\frac{d P}{d z}$ is the power dissipated in ohmic loss per unit length of the waveguide, and $P$ is the total power flow at $z$. This classical method has some disadvantages: it gives physically meaningless results at cut-off, where the attenuation constant tends to infinity, and losses associated with the evanescent modes are not taken into account. All these drawbacks can be overcome using the technique developed and called perturbation of boundary conditions [8], [9]. With this enhanced method at each analysis frequency, even at the modal cut-off frequency, both attenuation $\alpha$ and phase constant $\beta$ are computed taking positive real values $\left(e^{-\gamma z}=e^{-\alpha z} e^{-j \beta z}\right.$, being $\gamma$ the complex propagation constant), and losses associated to the evanescent modes are rigorously accounted for.

Next, we proceed to present the theoretical basics related to the proposed analysis technique considering first the TM modal problem. For finite but large conductivity values, the TM boundary condition on the waveguide contour $\left.E_{z 0}\right|_{\partial S}=0$ is not valid, because $E_{z}$ is not zero on the walls. From (1) and expressing the transverse magnetic field in terms of the longitudinal electric field [8], we have

$$
\begin{aligned}
\left.E_{z}\right|_{\partial S} \simeq & \left.\frac{(1+j)}{\sigma \delta} H_{0 l}\right|_{\partial S}=\left.\frac{(1+j)}{\sigma \delta} \frac{-j \omega \epsilon}{k_{c 0}^{2}} \frac{\partial E_{z 0}}{\partial n}\right|_{\partial S}= \\
& \left.\frac{(1-j)}{2} \frac{\mu_{c}}{\mu} \delta\left(\frac{\omega}{\omega_{c 0}}\right)^{2} \frac{\partial E_{z 0}}{\partial n}\right|_{\partial S}
\end{aligned}
$$

where $k_{c 0}=\omega_{c 0}^{2} \mu \epsilon$ is the modal lossless cut-off wavenumber, and $\mu$ the magnetic permeability of the medium which homogeneously fills the waveguide. So, the new proposed perturbed TM problem considering losses is thus

$$
\begin{gathered}
\left(\nabla_{t}^{2}+k_{c}^{2}\right) E_{z}=0 \\
\left.\left.E_{z}\right|_{\partial S} \simeq f_{T M} \frac{\partial E_{z 0}}{\partial n}\right|_{\partial S}
\end{gathered}
$$

with $f_{T M}=\frac{(1-j)}{2} \frac{\mu_{c}}{\mu} \delta\left(\frac{\omega}{\omega_{c 0}}\right)^{2}$.

In order to obtain the new complex propagation constant, the Green theorem in two dimensions can be employed:

$$
\begin{gathered}
\int_{S}\left(E_{z 0} \nabla_{t}^{2} E_{z}-E_{z} \nabla_{t}^{2} E_{z 0}\right) d S= \\
\left(k_{c 0}^{2}-k_{c}^{2}\right) \int_{S} E_{z 0} E_{z} d S=\oint_{\partial S}\left(E_{z} \frac{\partial E_{z 0}}{\partial n}\right) d l
\end{gathered}
$$

Since $f_{T M}$ is assumed to be a parameter with small value, it is consistent to approximate $E_{z}$ in the integral on the left hand size by its unperturbed value $E_{z 0}$, thus obtaining

$$
\gamma^{2}-\gamma_{0}^{2}=k_{c 0}^{2}-k_{c}^{2} \simeq f_{T M} \frac{\oint_{\partial S}\left|\frac{\partial E_{z 0}}{\partial n}\right|^{2} d l}{\int_{s}\left|E_{z 0}\right|^{2} d S}
$$

where $\gamma_{0}=\omega^{2} \mu \varepsilon-k_{c 0}^{2}$. Introducing the following parameter

$$
\xi_{T M}=\frac{f_{T M}}{2(1-j) \gamma_{0}} \frac{\oint_{\partial S}\left|\frac{\partial E_{z 0}}{\partial n}\right|^{2} d l}{\int_{S}\left|E_{z 0}\right|^{2} d S}
$$

the new complex propagation constant $\gamma$ considering losses is finally given by

$$
\gamma=\sqrt{\gamma_{0}\left(\gamma_{0}+2 \xi_{n}\right)-j 2 \xi_{n} \gamma_{0}} ; \quad n=T M, T E ;
$$

which is also valid for TE modes as it will be shortly demonstrated.

For the TE perturbed problem, we need to consider that an axial electric field component also appears on the surface, from (1) we have

$$
\left.\left.\mathbf{E}_{\|}\right|_{\partial S} \simeq\left(Z_{s} \frac{-j \gamma}{k_{c 0}^{2}} \frac{\partial H_{z 0}}{\partial l}\right)\right|_{\partial S} \hat{z}-\left.Z_{s} H_{z 0}\right|_{\partial S} \hat{l}
$$

Next, the normal component of the magnetic field associated to this axial electric field can be obtained by using the Maxwell equations [8]:

$$
\left.H_{n}\right|_{\partial S}=\left.\frac{j}{\mu_{c} \omega} \frac{\partial E_{z}}{\partial l}\right|_{\partial S}=\left.\frac{Z_{s} \gamma}{\mu_{c} \omega k_{c 0}^{2}} \frac{\partial^{2} H_{z 0}}{\partial l^{2}}\right|_{\partial S}
$$

Then, defining $f_{T E}=-(1-j) \frac{\delta}{2}$, we found that the perturbed TE problem considering losses is thus

$$
\begin{gathered}
\left(\nabla_{t}^{2}+k_{c}^{2}\right) H_{z}=0 \\
\left.\left.\frac{\partial H_{z}}{\partial n}\right|_{\partial S} \simeq f_{T E}\left(\omega^{2} \mu \epsilon \frac{\mu_{c}}{\mu} H_{z 0}-\frac{\gamma_{0}^{2}}{k_{c 0}^{2}} \frac{\partial^{2} H_{z 0}}{\partial l^{2}}\right)\right|_{\partial S}
\end{gathered}
$$

The last step in the formulation is to use the Green theorem in two dimensions, obtaining the following parameter for TE modes

$$
\begin{aligned}
\xi_{T E}= & \frac{-f_{T E}}{2(1-j) \gamma_{0}} \frac{\oint_{\partial S}\left(\omega^{2} \mu \epsilon \frac{\mu_{c}}{\mu}\left|H_{z 0}\right|^{2}-\frac{\gamma_{0}^{2}}{k_{c 0}^{2}} \frac{\partial^{2} H_{z 0}}{\partial l^{2}}\right) d l}{\int_{s}\left|H_{z 0}\right|^{2} d S}= \\
& \frac{\delta}{4 \gamma_{0}} \frac{\oint_{\partial S}\left(\omega^{2} \mu \epsilon \frac{\mu_{c}}{\mu}\left|H_{z 0}\right|^{2}+\frac{\gamma_{0}^{2}}{k_{c 0}^{2}}\left|\frac{\partial H_{z 0}}{\partial l}\right|^{2}\right) d l}{\int_{s}\left|H_{z 0}\right|^{2} d S}
\end{aligned}
$$

where the last expression is obtained after some algebraic manipulation. Finally, the TE complex propagation constant can be obtained using again (9).

\section{Results}

Note that in all the next figures we indicate with method 1 the results obtained by the classical power-loss method, and with method 2 the results obtained by the proposed technique. First of all, in order to validate our theory, we have performed the modal analysis of a WR-75 rectangular waveguide ( $a=$ $19.05 \mathrm{~mm}$ and $b=9.525 \mathrm{~mm}$ ) with a finite conductivity value of $\sigma=5.8 \cdot 10^{7} \mathrm{~S} / \mathrm{m}$. Fig. 1(a) shows the attenuation constant of the first WR-75 waveguide modes as function of frequency computed with method 1 , with method 2 and the commercial software HFSS ${ }^{1}$. Note that method 2 does not fail at the cut-off frequency in contrast with classical method 1 (infinite value

\footnotetext{
${ }^{1}$ www.ansoft.com/products/hf/hfss 12
} 


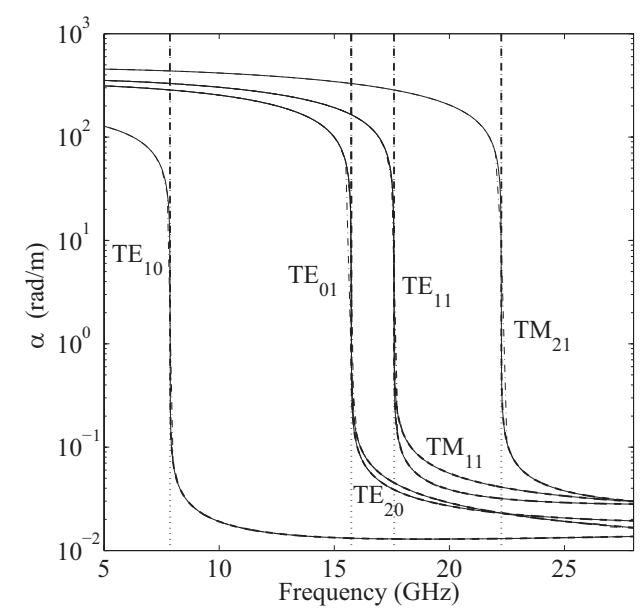

(a)

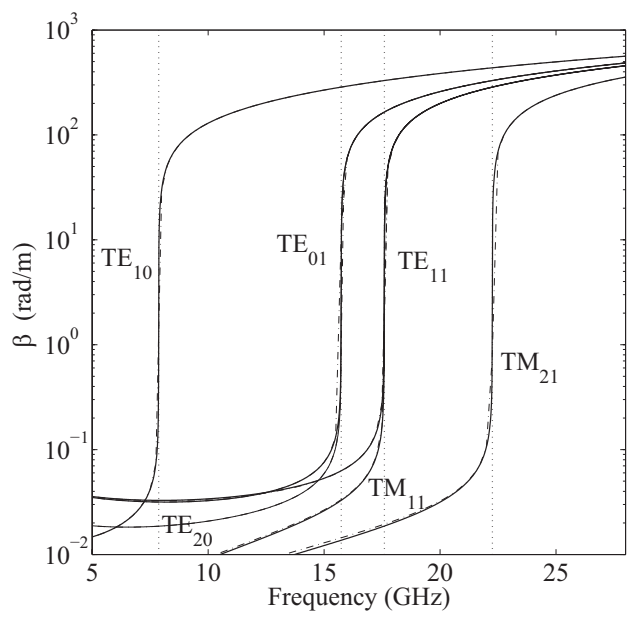

(b)

Fig. 1. Attenuation (a) and phase constants (b) for the first WR75 rectangular modes computed with method 1 (dashed lines, only in (a)), method 2 (solid line) and HFSS data (dashdot line). The vertical dotted lines indicate the cut-off frequency of the different modes in the lossless case.

for $\alpha$ ). In Fig. 1(b) we also present the phase constant for the same modes computed with method 2 and HFSS data. A very good agreement between our results and those from Ansoft can be observed.

With the aim of verifying the accuracy of the previous results, Fig. 2 successfully compares the attenuation versus phase constant of the first WR-28 $(a=7.112 \mathrm{~mm}$ and $b=3.556 \mathrm{~mm}$ ) rectangular mode for a conductivity range between $10^{5}$ and $5.8 \cdot 10^{7} \mathrm{~S} / \mathrm{m}$, computed at frequencies close to the cut-off $(21.076 \mathrm{GHz})$. The points are also computed with HFSS.

Once the proposed theory has been validated with several

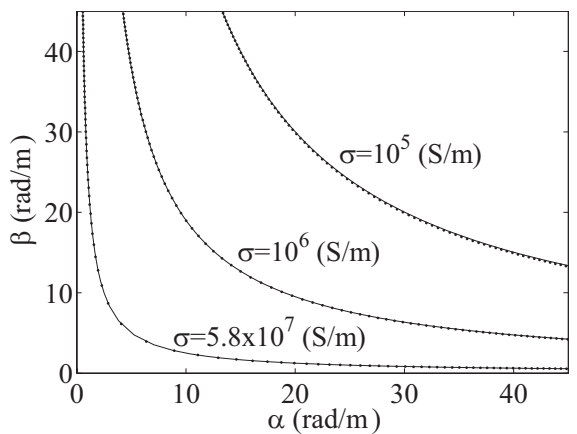

Fig. 2. Phase constant versus attenuation of the first WR-28 rectangular mode at frequencies close to cut-off $(21.076 \mathrm{GHz})$, for different conductivities and computed with method 2 (solid lines) and with HFSS (points).

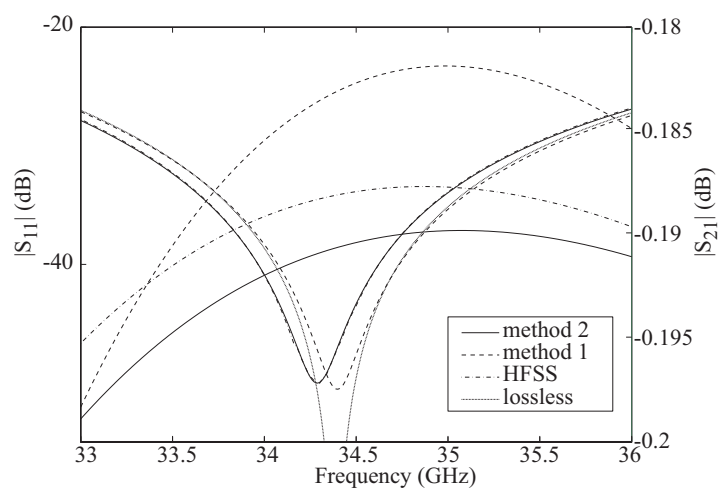

Fig. 3. Scattering parameters of an input/output WR-28 waveguide ( $a=7.112 \mathrm{~mm}$ and $b=3.556 \mathrm{~mm}$ ) coupled with a central iris of dimensions: $a=5.69 \mathrm{~mm}, b=2.845 \mathrm{~mm}$ and length $l=2 \mathrm{~mm}$.

benchmark tests, its direct application to the analysis of passive rectangular waveguide structure is faced. For this purpose, we have integrated the enhanced technique within a CAD software package based on the Integral-Equation method (IE) [10]. As first example, we have simulated a very simple case: an input/output WR-28 waveguide coupled with a central iris of dimensions $a=5.69 \mathrm{~mm}, b=2.845 \mathrm{~mm}$ and length $l=2 \mathrm{~mm}$. We have assumed a low finite conductivity value of $\sigma=10^{5} \mathrm{~S} / \mathrm{m}$. In Fig. 3 the simulated reflection and transmission coefficients of this simple structure are compared with the numerical data also provided by HFSS, where also only propagation losses effects (longitudinal walls) have been considered. It can be seen clearly, due to the low conductivity value, a phase shift of the magnitude of $S_{11}$ appears. This shift, caused by a variation of the value of $\beta$, is not predicted by the classical method 1 .

Finally, making use of the CAD software package implemented, we have designed a 2-pole symmetric rectangular bandpass filter for operation at $30 \mathrm{GHz}$ and with a bandwidth of $600 \mathrm{MHz}$. Input/output filter ports and cavities are in WR28 standard rectangular waveguides. To study the propagation loss effect, in this case a finite conductivity value of $10^{6} \mathrm{~S} / \mathrm{m}$ has been assumed. The simulated scattering parameters of such 


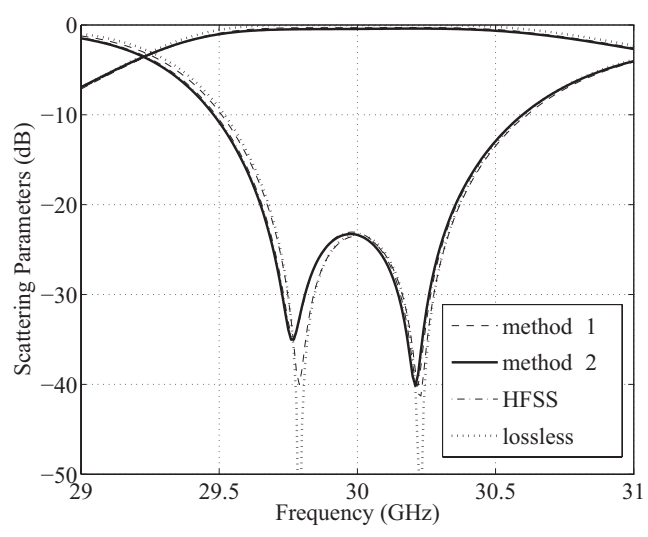

Fig. 4. Scattering parameters of a 2-pole symmetric bandpass filter. Dimensions: input/output ports and cavities are in WR-28 waveguides; the cavity length is $11.457 \mathrm{~mm}$. Dimensions of the input/output coupling irises are $a_{1}=4.9 \mathrm{~mm}, b_{1}=2.85 \mathrm{~mm}$; central iris with $a_{2}=4.442 \mathrm{~mm}, b_{2}=1.259 \mathrm{~mm}$. All irises of length $2 \mathrm{~mm}$.

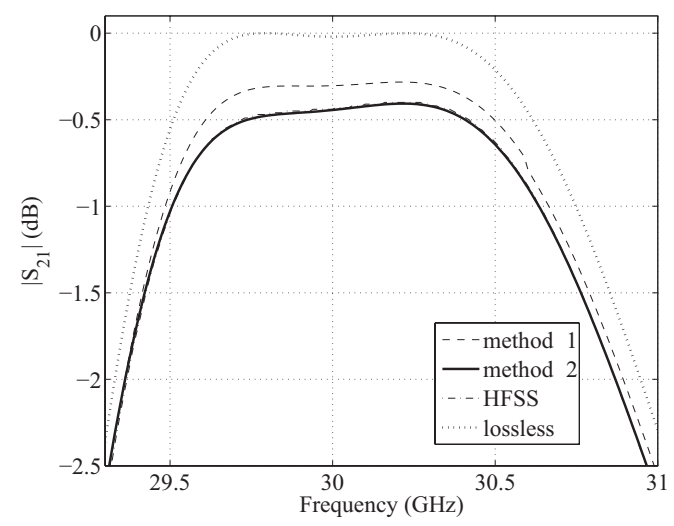

Fig. 5. Detailed view of the magnitude of the transmission coefficient $\left(S_{21}\right)$ for the 2-pole symmetric bandpass filter.

a structure are shown in Fig. 4, where they are successfully compared with the numerical data provided by HFSS software (dashdot line). Dashed and dotted lines represent, respectively, the results obtained with method 1 and considering perfect conductor walls. As can be seen from Fig. 4, a small deviation of the filter response appears with respect to the ideal case. This shift, which can be critical for narrow-band applications, is not considered either by the classical method 1. In Fig. 5 a detailed view of the magnitude of the transmission coefficient $\left(S_{21}\right)$ is shown. An identical level of $0.5 \mathrm{~dB}$ insertion loss is computed by our theory and HFSS results (where a value of 0.001 maximum delta $\mathrm{S}$ has been used as convergence criterion). With method 1 this level is higher because all modes in the rectangular irises are under cut-off, so losses in all these irises are not taken into account. Such results (for both methods) were obtained using 20 accessible modes, 100 basis functions, and 400 kernel terms in the IEs related to the solution of each discontinuity [10].

\section{CONCLUSION}

In this paper, a novel full-wave method for the computation of propagation losses in metallic waveguide structures is presented. Following this technique, the propagation losses effects are accurately predicted using a perturbation of the boundary conditions on the waveguide contour combined with an integral-equation analysis technique. With this new approach the drawbacks of the power method can be overcome, and losses associated to the evanescent modes can also be taken into account. The new proposed theory has been extensively verified through several examples of practical interest. First, the complex propagation constants of some rectangular waveguide modes have been successfully computed. Next a simple input/output WR-28 waveguide coupled by a central iris has been considered, and the scattering parameters obtained using the developed method and numerical data provided by a commercial software have been successfully compared. Finally, the new modal analysis technique has been used together with a CAD software package for design purposes. Successful results for a 2-pole symmetric bandpass filter used for $\mathrm{Ka}$ band applications, have proved the accuracy of the proposed method.

\section{ACKNOWLEDGEMENT}

This work has been supported by Ministerio de Educación y Ciencia, Spanish Government, under the Grant JC20090221 and the coordinated R\&D project TEC2010-21520-C04, by University of Alicante under the project GRE10-22, as well as by the European COST Office under Action IC-0803 "RF/Microwave Communication Subsystems for Emerging Wireless Technologies (RFCSET)”.

\section{REFERENCES}

[1] I. Hunter, L. Bilonet, B. Jarry, and P. Guillon, "Microwave filterapplications and technology," IEEE Trans. Microwave Theory Tech., vol. 50, no. 3, pp. 794-805, Mar. 2002.

[2] V. Miraftab and M. Yu, "Generalized lossy microwave filter coupling matrix syntesis and design using mixed technologies," IEEE Trans. Microwave Theory Tech., vol. 56, no. 12, pp. 3016-3027, 2008.

[3] M. Oldoni, G. Macchiarella, G. G. Gentili, and C. Ernst, "A new approach to the synthesis of microwave lossy filters," IEEE Trans. Microwave Theory Tech., vol. 58, no. 5, pp. 1222-1229, May 2010.

[4] R. E. Collin, Field Theory of Guided Wave. New York: McGraw-Hill, 1960.

[5] J. Justincic, "A general power loss method for attenuation of cavities and waveguides," IEEE Trans. Microwave Theory Tech., vol. 11, no. 1, pp. 83-87, 1963.

[6] A. Melloni and G. Gentili, "Modellization of losses in $\mathrm{TE}_{011}$ mode waveguide bandpass filter," IEEE Trans. Microwave Theory Tech., vol. 43, no. 11, pp. 2642-2644, Nov. 1995.

[7] J. Hueso, S. Cogollos, B. Gimeno, V. Boria, A. Vidal, M. Taroncher, H. Esteban, and M. Guglielmi, "Accurate consideration of metal losses in planar waveguide junctions using an efficient integral equation technique," in IEEE MTT-S Int. Microwave Symp. Dig., 2004, pp. 14111414.

[8] J. D. Jackson, Classical Electrodynamics. New York: J. Wiley \& Sons, 2001

[9] W. Wilcox, Macroscopic Electrodynamics. Baylor University, 2005.

[10] G. Gerini, M. Guglielmi, and G. Lastoria, "Efficient integral equation formulations for admittance or impedance representation of planar waveguide junctions," in IEEE MTT-S Int. Microwave Symp. Dig., vol. 3, 1998, pp. 1747-1750. 